\title{
The COMPUTATION OF ISOTROPIC VECTORS
}

\author{
G. Meurant \\ http://gerard.meurant.pagesperso-orange.fr/ \\ Paris, France \\ gerard.meurant@gmail.com
}

We show how to compute isotropic vectors for matrices with real or complex entries. These are unit vectors $b$ satisfying $b^{*} A b=0$ where the $*$ denotes the conjugate transpose. For real matrices, the algorithm uses only the eigenvectors of the symmetric part of $A$ corresponding to the extreme eigenvalues. For complex matrices, we first use the eigenvalues and eigenvectors of the Hermitian matrix $K=\left(A-A^{*}\right) / 2 i$. This works in many cases. In case of failure, we use the eigenvectors of the Hermitian part $H$ or a combination of eigenvectors of $H$ and $K$. We give some numerical experiments comparing our algorithm with those proposed in [2] and [1]. In many cases our algorithm use only one computation of eigenvectors whence the other algorithms use at least two computations of eigenvectors.

\section{References}

[1] R. Carden, A simple algorithm for the inverse field of values problem, Inverse Problems, v 25 (2009), pp. 1-9.

[2] C. Chorianopoulos, P. Psarrakos and F. Uhlig, A method for the inverse numerical range problem, Elec. J. Linear Alg., v 20 (2010), pp. 198-206. 